\title{
MR
}

\section{CYP2C19 polymorphisms in acute coronary syndrome patients undergoing clopidogrel therapy in Zhengzhou population}

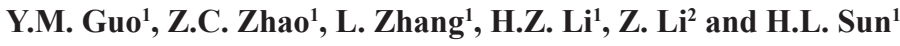 \\ ${ }^{1}$ Department of Cardiovascular Internal Medicine, \\ Zhengzhou Central Hospital Affiliated to Zhengzhou University, \\ Zhengzhou, China \\ ${ }^{2}$ Institute of Medicine, Central South University, Changsha, China \\ Corresponding author: Z.C. Zhao \\ E-mail: zhichenzhao@yeah.net \\ Genet. Mol. Res. 15 (2): gmr.15028012 \\ Received November 11, 2015 \\ Accepted January 15, 2016 \\ Published May 25, 2016 \\ DOI http://dx.doi.org/10.4238/gmr.15028012
}

\begin{abstract}
The goal of this study was to explore the polymorphisms of CYP2C19 (CYP2C19*2, CYP2C19*3) in patients with acute coronary syndrome (ACS) undergoing percutaneous coronary intervention (PCI) on clopidogrel therapy in Zhengzhou city for guidance on clinical medication and reduction in the incidence of thromboembolic events. Two hundred and thirty-four ACS patients undergoing PCI were included in the study, including 171 males (average age $=64.13 \pm 12$ years) and 63 females (average age $=67.86 \pm 10.20$ years). Pyrosequencing analysis detected CYP2C19*2/*3 genotypes, which were divided into wild-type homozygous $\mathrm{C} / \mathrm{C}$, mutant heterozygous $\mathrm{C} / \mathrm{T}$, and mutant homozygous T/T. This study further explored the relationship between CYP2C19 polymorphisms and clopidogrel resistance in ACS patients. Gene frequencies of $\mathrm{C} / \mathrm{C}, \mathrm{C} / \mathrm{T}$, and T/T for CYP2C19*2 were 39.74, 50, and $10.26 \%$, respectively, while the frequencies of $\mathrm{C} / \mathrm{C}, \mathrm{C} / \mathrm{T}$, and $\mathrm{T} / \mathrm{T}$ for CYP2C19*3 were 94.02, 5.55, and 0.43\%, respectively. According to platelet aggregation analysis, 203 cases normally responded to
\end{abstract}


clopidogrel (86.8\%) and 31 cases were clopidogrel resistant (13.2\%). There was a correlation between gender and genotype distribution but none between age and genotype. In addition, patients with clopidogrel resistance were treated with ticagrelor antiplatelet therapy instead of clopidogrel, and only 1 case in all patients suffered thrombotic events during a 3-12 month follow-up. In conclusion, CYP2C19*2/*3 polymorphisms may be associated with clopidogrel resistance. Wildtype homozygote and single mutant heterozygote of CYP2C19*2/*3 can be given a normal dose of clopidogrel, while carriers with single mutant homozygote or double mutant heterozygote require ticagrelor antiplatelet therapy as an alternative.

Key words: Clopidogrel; Polymorphism; Coronary heart disease

\section{INTRODUCTION}

As life styles change, the prevalence of coronary heart disease in China has increased year by year, and interventional therapy has become an important means to alleviate the symptoms of angina pectoris. Antiplatelet therapy is an important method for the prevention of thrombosis and in-stent restenosis before and after surgery. The combination of aspirin and clopidogrel is the standard medication of anti-platelet therapy for patients with acute coronary syndrome (ACS) undergoing percutaneous coronary intervention (PCI), but a number of patients are resistant to clopidogrel. As a prodrug, clopidogrel cannot play an anti-platelet role unless it exerts activity after enzymatic metabolism. Therefore, more attention has been focused on the association between the CYP2C19*2/*3 polymorphisms that affect enzymatic metabolism and clopidogrel resistance.

Clopidogrel exerts its inhibition on platelet aggregation after transformation with the help of the cytochrome P450 (CYP450) enzyme in vivo, which can effectively reduce the risk of intra-arterial and postoperative in-stent thrombosis. CYP2C19 is an important member of the CYP450 isoenzyme family and is involved in the catalytic metabolism of endogenous substrates, environmental pollutants, and about $2 \%$ of clinical drugs. Chinese and international studies have confirmed that the CYP2C19 gene polymorphism is associated with clopidogrel metabolism. Moreover, due to the genetic polymorphisms, there are obvious individual and racial differences in CYP2C19 enzyme activity, which has a serious impact on the clinical efficacy and safety of clopidogrel. Therefore, it is important to understand the genetic polymorphisms of CYP2C19 for the implementation of individual anti-platelet therapy. In this study, the distribution of CYP $2 \mathrm{C} 19 * 2 / * 3$ gene polymorphisms in patients with ACS undergoing PCI in Zhengzhou, China was examined.

\section{MATERIAL AND METHODS}

\section{Subjects}

A total of 234 ACS patients undergoing PCI in Zhengzhou consecutively registered from October 2013 to August 2014 were admitted to the Zhengzhou Central Hospital Affiliated 
to Zhengzhou University. They were 39-90 years old with the average age being $65.13 \pm 11.64$. The group included 171 males (average age $=64.13 \pm 12$ ) and 63 females (average age $=67.86$ \pm 10.20 ). This study was conducted in accordance with the declaration of Helsinki and with approval from the Ethics Committee of Zhengzhou Central Hospital Affiliated to Zhengzhou University. Written informed consent was obtained from all participants. The inclusion criteria were: 1) Han patients with ACS undergoing PCI; 2) born and currently lived in Zhengzhou; and 3) had not taken clopidogrel before admission. The exclusion criteria were: 1) allergy and intolerance to clopidogrel; 2) severe liver or renal dysfunction or abnormal coagulation function; 3) perioperative gastrointestinal bleeding or active bleeding history within the last 30 days; 4) platelet count $<100 \times 10^{9} / \mathrm{L}$ or $>300 \times 10^{9} / \mathrm{L}$ and white blood cell count $<4 \times 10^{9} / \mathrm{L}$ or $>10 \times 10^{9} / \mathrm{L}$ in hospital blood test; and 5) minority patients. For surgery, 1-2 rapamycineluting stents were implanted into all patients with release at 12-14 atm and were expanded with balloon post-dilatation at 12-16 atm to make in-stent residual stenosis less than $10 \%$.

\section{CYP2C19 genetic testing}

Two-milliliter blood samples were collected before PCI and was placed into an EDTA anticoagulated tube. Blood was preserved at $4{ }^{\circ} \mathrm{C}$ to analyze genotypes within $24 \mathrm{~h}$.

\section{Pyrosequencing reaction}

PCR amplification product $(15 \mu \mathrm{L})$ was transferred onto 96-well plates with $38 \mu \mathrm{L}$ binding buffer (Qiagen, Dusseldorf, Germany) added to each PCR reaction system (Table 1). Next, the reaction was placed on a multifunction oscillator (Eppendorf, Hamburg, Germany) at $2000 \mathrm{rpm}$ with $2 \mu \mathrm{L}$ Streptavidin Sepharose beads (GE, Beijing, China) and incubated for $10 \mathrm{~min}$. After Ultrapure water, $70 \%$ ethanol, denaturation buffer $(0.2 \mathrm{M} \mathrm{NaOH})$ and washing buffer (Qiagen) were consecutively added in 4 pools to the Vacuum Prep Workstation (Qiagen). The vacuum pump was turned on and the vacuum prep tool was rinsed in ultrapure water for $30 \mathrm{~s}$ and inserted onto 96-well plates to absorb Sepharose beads. The vacuum prep tool was washed in 70\% ethanol for $5 \mathrm{~s}$, denatured in denaturation buffer for $8 \mathrm{~s}$ (only biotinlabeled single-stranded DNA bound to Sepharose beads), and rinsed in washing buffer for $10 \mathrm{~s}$. As the vacuum pump was turned off, the vacuum prep tool was placed onto PyroMark Q24 pyrosequencing reaction plates (Qiagen) with $25 \mu \mathrm{L}$ annealing buffer (Qiagen) and 1.5 $\mu \mathrm{L}(10 \mu \mathrm{M})$ sequencing primer already added to each well. After gentle friction, Sepharose beads were fully released. The reaction plate was placed at $80^{\circ} \mathrm{C}$ for $2 \mathrm{~min}$ and cooled to room temperature. The test procedure was set according to the manufacturer instructions for Q24 PyroMark. After enzyme mixture (E), fluorescent substrate (S), and four kinds of dNTPs in PyroMark ${ }^{\mathrm{TM}}$ Gold Q96 Reagents kit (Qiagen) were added to the reagent chamber, the reaction plate was detected in the detection chamber. Amplification and sequencing primers used in the pyrosequencing reaction are shown in Table 1.

\section{Detection method of platelet aggregation}

After dual antiplatelet therapy of aspirin and clopidogrel for 1 week, $2 \mathrm{~mL}$ blood was collected from each patient into an EDTA anticoagulated tube. Patients could not ingest milk, 
soymilk, or other high fat foods the day before blood sampling for the prevention of reducing the transmittance artificially. The ADP-induced platelet aggregation method was applied for the determination of platelet aggregation within $30 \mathrm{~min}$. To prepare, $1 \mathrm{~mL}$ ADP buffer was added into ADP powder and mixed then another $1 \mathrm{~mL}$ ADP buffer was added and mixed. This ADP solution $(10 \mathrm{~mL})$ was divided into several low-dose aliquots. For dosage, each patient received $10 \mu \mathrm{L}$ (final concentration $=5 \mu \mathrm{M}$ ). The Platelet-Poor Plasma (PPP) sample was centrifuged at $2500 \mathrm{rpm}$ for $10 \mathrm{~min}$, while Platelet-Rich Plasma (PRP) sample was centrifuged at $800 \mathrm{rpm}$ for $10 \mathrm{~min}$. For detection, $300 \mu \mathrm{L}$ PPP plasma (without test beads) was added into the test cup and placed into the test channel for sampling in the PPP state. In addition, $300 \mu \mathrm{L}$ PRP plasma (with test beads) was added into the test cup, transferred into the test channel, and rewarmed at $37^{\circ} \mathrm{C}$ for $1 \mathrm{~min}$. The device was then turned on. Immediately, $10 \mu \mathrm{L}$ inducer (ADP) was added into the cup bottom with a pipette. An automatic test was performed and the results recorded.

Table 1. Amplification and sequencing primers in pyrosequencing reaction.

\begin{tabular}{l|l|l}
\hline Primer & Primer sequence $\left(5^{\prime} \rightarrow 3^{\prime}\right)$ & Explanation \\
\hline $61-\mathrm{F}$ & TGTGTTTCTCCCTTCTCAGGATTC & 5' end labeled with biotin, PCR amplification primers \\
\hline $61-$ & TACTGGTCCCTCATTGCACTGT & PCR amplification primers \\
\hline $61-$ pyroS & ATATTCTCGACACAGCAG & Pyrosequencing primers \\
\hline $1213-\mathrm{F}$ & ACTGGTGGAGTATTTGATAGTGTA & $5^{\prime}$ end labeled with biotin, PCR amplification primers \\
\hline $1213-\mathrm{R}$ & CCTCTATTGTTGGATCATATTCGT & PCR amplification primers \\
\hline $1213-$ pyroS & AGCTCCAACTACCACAA & Pyrosequencing primers \\
\hline
\end{tabular}

\section{Statistical analysis}

All statistical analyses were performed using SPSS 13.0 (SPSS Inc., Chicago, IL, USA) and data are reported as means \pm standard deviation. Comparison of averages was analyzed using the Student $t$-test between the two groups. The Pearson chi-square test was applied for correlation analysis. $\mathrm{P}<0.05$ was considered statistically significant.

\section{RESULTS}

\section{Generation information}

A total of 234 ACS patients undergoing PCI in Zhengzhou were consecutively registered as they were admitted to the hospital. They were 39 to 90 years old with an average age of $65.13 \pm 11.64$ years (Table 2). The group comprised 171 males (average age $=64.13 \pm$ 12 years) and 63 females (average age $=67.86 \pm 10.20$ years). There were 122 patients with hypertension and 89 patients with diabetes.

\section{Table 2. General information on selected patients.}

\begin{tabular}{l|c|c|c}
\hline Gender & $\mathrm{N}$ & Age (years) (means \pm SD) & BMI \\
\hline Female & 171 & $64.13 \pm 12.00$ & $26.59 \pm 4.56$ \\
\hline Male & 63 & $67.86 \pm 10.20$ & $24.32 \pm 6.23$ \\
\hline
\end{tabular}




\section{Genotype frequencies of the polymorphic loci of CYP2C19*2 and CYP2C19*3}

Statistical results showed that genotype frequencies of mutant heterozygote $(50 \%)$ and wild-type homozygote $(94.02 \%)$ of CYP2C19*2 were the highest, while the mutation rate of CYP2C19*3 was the lowest $(0.43 \%$; Table 3$)$.

Table 3. Frequency of each locus of CYP2C19*2 and CYP2C19*3.

\begin{tabular}{l|c|c|c|c|c|c}
\hline \multirow{2}{*}{} & \multicolumn{3}{|c|}{ CYP2C19*2 } & \multicolumn{3}{c}{ CYP2C19*3 } \\
\cline { 2 - 7 } & $\mathrm{C} / \mathrm{C}$ & $\mathrm{C} / \mathrm{T}$ & $\mathrm{T} / \mathrm{T}$ & $\mathrm{C} / \mathrm{C}$ & $\mathrm{C} / \mathrm{T}$ & $\mathrm{T} / \mathrm{T}$ \\
\hline $\mathrm{N}$ & 93 & 117 & 24 & 220 & 13 & 1 \\
\hline Frequency (\%) & 39.74 & 50 & 10.26 & 94.02 & 5.55 & 0.43 \\
\hline
\end{tabular}

$\mathrm{C} / \mathrm{C}=$ Wild-type homozygote (fast metabolism of clopidogrel). $\mathrm{C} / \mathrm{T}=$ Mutation heterozygote (intermediate metabolism of clopidogrel). $\mathrm{T} / \mathrm{T}=$ Mutation homozygote (low metabolism of clopidogrel).

\section{Genotype distribution of CYP2C19*2/*3 in patients}

Statistical results showed that genotype frequencies of CYP2C19*2 C/T and CYP2C19*3 $\mathrm{C} / \mathrm{C}$ were the highest, accounting for $47.01 \%(\mathrm{~N}=110)$, while the frequency of the double mutant homozygote of CYP2C19*2/*3 was 0 (Table 4 ).

\begin{tabular}{|c|c|c|c|c|c|c|c|}
\hline \multirow[t]{2}{*}{ Gender } & \multicolumn{6}{|c|}{ Cases } & \multirow[t]{2}{*}{$\mathrm{N}$} \\
\hline & $\mathrm{CC} / \mathrm{CC}^{1}$ & $\mathrm{CT} / \mathrm{CC}^{2}$ & $\mathrm{TT} / \mathrm{CC}^{3}$ & $\mathrm{CC} / \mathrm{CT}^{4}$ & $\mathrm{CC} / \mathrm{TT}^{5}$ & $\mathrm{CT} / \mathrm{CT}^{6}$ & \\
\hline Female & 67 & 83 & 15 & 1 & 1 & 4 & 171 \\
\hline Male & 20 & 27 & 9 & 5 & 0 & 2 & 63 \\
\hline Total & 87 & 110 & 24 & 6 & 1 & 6 & 234 \\
\hline Proportion (\%) & 37.18 & 47.01 & 10.26 & 2.56 & 0.43 & 2.56 & 100 \\
\hline \multicolumn{8}{|c|}{$\begin{array}{l}{ }^{1} \mathrm{CC} / \mathrm{CC}=\mathrm{CYP} 2 \mathrm{C} 19 * 2(\mathrm{C} / \mathrm{C}), \mathrm{CYP} 2 \mathrm{C} 19 * 3(\mathrm{C} / \mathrm{C}), \mathrm{CYP} 2 \mathrm{C} 19 * 2 / 3 \text { wild-type homozygote. }{ }^{2} \mathrm{CT} / \mathrm{CC}=\mathrm{CYP} 2 \mathrm{C} 19 * 2 \\
(\mathrm{C} / \mathrm{T}), \mathrm{CYP} 2 \mathrm{C} 19 * 3(\mathrm{C} / \mathrm{C}), \mathrm{CYP} 2 \mathrm{C} 19 * 2 \text { single mutation heterozygote. }{ }^{3} \mathrm{TT} / \mathrm{CC}=\mathrm{CYP} 2 \mathrm{C} 19 * 2(\mathrm{~T} / \mathrm{T}), \mathrm{CYP} 2 \mathrm{C} 19 * 3 \\
(\mathrm{C} / \mathrm{C}), \mathrm{CYP} 2 \mathrm{C} 19 * 2 \text { single mutation homozygote. }{ }^{4} \mathrm{CC} / \mathrm{CT}=\mathrm{CYP} 2 \mathrm{C} 19 * 2(\mathrm{C} / \mathrm{C}), \mathrm{CYP} 2 \mathrm{C} 19 * 3(\mathrm{C} / \mathrm{T}), \mathrm{CYP} 2 \mathrm{C} 19 * 3 \\
\text { single mutation heterozygote. }{ }^{5} \mathrm{CC} / \mathrm{TT}=\mathrm{CYP} 2 \mathrm{C} 19 * 2(\mathrm{C} / \mathrm{C}), \mathrm{CYP} 2 \mathrm{C} 19 * 3(\mathrm{~T} / \mathrm{T}), \mathrm{CYP} 2 \mathrm{C} 19 * 3 \text { single mutan } \\
\text { homozygote. }{ }^{6} \mathrm{CT} / \mathrm{CT}=\mathrm{CYP} 2 \mathrm{C} 19 * 2(\mathrm{C} / \mathrm{T}), \mathrm{CYP} 2 \mathrm{C} 19 * 3(\mathrm{C} / \mathrm{T}), \mathrm{CYP} 2 \mathrm{C} 19 * 2 / 3 \text { double mutant heterozygote. }\end{array}$} \\
\hline
\end{tabular}

\section{Platelet aggregation of each genotype}

The platelet aggregation of each genotype was compared, and the results showed that there were no significant differences in $\mathrm{CC} / \mathrm{CC}, \mathrm{CT} / \mathrm{CC}, \mathrm{CC} / \mathrm{CT}(\mathrm{P}>0.05)$ and in TT/CC, $\mathrm{CC} / \mathrm{TT}, \mathrm{CT} / \mathrm{CT}(\mathrm{P}>0.05)$. Therefore, CC/TT, TT/CC, CT/CT were considered as clopidogrel resistant and theses carriers needed ticagrelor antiplatelet therapy instead of clopidogrel therapy (Table 5).

\section{Table 5. Platelet aggregation of each genotype.}

\begin{tabular}{l|c|c}
\hline Genotype & $\mathrm{N}$ & Mean platelet aggregation \\
\hline $\mathrm{CC} / \mathrm{CC}$ & 87 & 19 \\
\hline $\mathrm{CT} / \mathrm{CC}$ & 110 & 16 \\
\hline $\mathrm{TT} / \mathrm{CC}$ & 24 & 16 \\
\hline $\mathrm{CC} / \mathrm{CT}$ & 6 & 28 \\
\hline $\mathrm{CC} / \mathrm{TT}$ & 1 & 32 \\
\hline $\mathrm{CT} / \mathrm{CT}$ & 6 & 31 \\
\hline
\end{tabular}




\section{Analysis of the ratio of clopidogrel sensitive group and clopidogrel resistant group}

According to the platelet aggregation results, 203 patients had normal metabolism of clopidogrel (86.8\%) while 31 cases were clopidogrel resistant (13.2\%). Timely identification of patients with clopidogrel resistance can reduce cardiovascular thrombotic events and the mortality and morbidity of coronary heart disease.

\section{Correlation analysis of genotype with gender and age}

The analysis showed that the genotype of CYP2C19*2/*3 was correlated with gender $(\mathrm{P}=0.028)$. The frequencies of wild-type homozygous CYP $2 \mathrm{C} 19 * 2 / * 3$ and mutant heterozygous CYP2C19*2 in males was higher (28.63 and $35.47 \%$, respectively), which might be related to the high proportion of males in registered patients. Moreover, there was no correlation between genotype and age $(\mathrm{P}=0.144)$.

\section{Comparison of the number of stents in both groups}

The average number of stents in the clopidogrel group was $1.34 \pm 1.13$, while the number in the ticagrelor group was $1.35 \pm 1.18$. The difference between the two groups was not statistically significant $(\mathrm{P}>0.05)$.

\section{Follow-up}

A total of 203 patients had a normal response to clopidogrel while 31 patients were resistant to clopidogrel. In addition, patients with clopidogrel resistance were treated with ticagrelor antiplatelet therapy instead of clopidogrel. During a 3-12 month follow-up, the primary endpoint events were: patient was hospitalized because of acute myocardial infarction, in-stent restenosis and angina pectoris, or death. One patient who was wild-type homozygous of CYP2C19*2/*3 in the clopidogrel sensitive group suffered from acute myocardial infarction after PCI.

\section{DISCUSSION}

Activation and aggregation of platelets plays an important role in arterial thrombosis after ACS and PCI. Oral anticoagulant therapy is the key treatment of ACS patients undergoing PCI. As a prodrug, clopidogrel is metabolized into active products in two steps in the liver CYP450 system after absorption. In the second oxidation step, 44.9 and $20.6 \%$ of the roles are mediated by the proteins encoded by the CYP2C19 gene (Kazui et al., 2010). The CYP2C19 gene is located in chromosome 10 in humans and includes 9 exons and 5 introns. It encodes proteases in liver microsomes, so this gene plays an important role in the transformation of clopidogrel in vivo. The gene encoding CYP2C19 shows polymorphic expression in the human body and the mutants are CYP2C19*2, CYP2C19*3, CYP2C19*4 and CYP2C19*5. $\mathrm{CYP} 2 \mathrm{C} 19 * 2$ is the major mutant while CYP2C19*3 is relevant within certain ethnic groups.

Recent research has shown that changes in the activity of drug-metabolizing enzymes resulting from genetic mutations is one of the root causes of different metabolic ability of the same drug in individuals (Severino and Del Zompo, 2004). CYP2C19 gene polymorphisms 
lead to individual differences in CYP2C19 enzyme activity, thereby causing inter-individual differences in metabolic capacity of clopidogrel (Liboredo and Pena, 2014; Sun et al., 2015). Foreign studies have indicated that the polymorphisms of CYP2C19 have obvious racial differences, as the incidence of CYP2C19*2 is 17,15 , and $30 \%$ and the incidence of CYP2C19*3 is 0.4, 4, and 5\% in black Americans, Caucasians and Chinese, respectively (Zhou et al., 2009). The incidence of chronic metabolism is 3,15-17, and $10-25 \%$ in Caucasians, Chinese, and Asians, respectively (Zhou et al., 2009). Moreover, the incidence of CYP2C19*2, CYP2C19*3, and chronic metabolism in Chinese (12-16\%) is much higher than in Caucasians (2-3\%) and Africans (4\%) (Wedlund, 2000). At present, there are at least 25 loci for CYP2C19 polymorphisms, and it has been observed that clopidogrel therapy for carriers of CYP $2 \mathrm{C} 19 * 17$ can significantly reduce the incidence of cardiovascular events when compared to carriers of CYP2C19*1. However, most current studies often focus on CYP2C19*2 and CYP2C19*3, which can diminish the efficacy of clopidogrel. These two alleles are the most common in Asians and their incidences are 29-35 and 2-9\%, respectively (Scott et al., 2013). However, their incidence in Chinese patients can be as high as 52.2 and 9.6\%, respectively (Zhang et al., 2013). Carriers of CYP2C19*2 and *3, referred to as having CYP2C19 chronic metabolism, present the slow activation rate of clopidogrel, resulting in decreased anti-platelet activity due to less active metabolites. The risk of in-stent thrombosis in patients with CYP2C19*2 was increased, which results in a high rate of disability and mortality; therefore, ticagrelor antiplatelet therapy is suggested for patients with CYP2C19*2 and *3. Collet et al. (2009) showed that the CYP2C19*2 polymorphism was an independent predictor of cardiovascular events and was a major determinant of prognosis in the study of 259 young patients (aged $<45$ years) having suffered from myocardial infarction and currently undergoing clopidogrel therapy. Hou et al. (2014) showed that CYP2C19*2 might be closely related to clopidogrel resistance in a meta-analysis of 2331 patients. However, current research on these polymorphisms is mainly conducted in Europeans and Americans, and the mechanism of their action needs to be further studied. In addition, there is no direct evidence that CYP2C19 is causally related with clopidogrel biotransformation, so some studies do not show the association between CYP2C19 gene polymorphisms and clopidogrel resistance. At present, CYP2C19 has been found to have at least 35 alleles (Zhang and Zhu, 2011). The mutant alleles affect the activity and expression of the enzyme. For example, CYP2C19*1 (wild-type or strong metabolism) encodes the normal enzyme and CYP2C19*2 (weak metabolism) is the most common enzyme-deficient allele. The CYP2C19*2 polymorphism reduces the active metabolic conversion of clopidogrel so the pharmacological metabolism of the body is weakened, which results in a low response to clopidogrel. Jeong et al. (2011) showed that $60.9 \%$ of patients had at least one locus mutation and the risk of high platelet reactivity and cardiovascular events were related to the number of variable loci in an East Asian population. There was no significant difference between carriers with one loss-of-function (LOT) gene and carriers with no loss-of-function gene, while the risks of platelet reactivity and cardiovascular events in carriers with 2 LOT genes was significantly increased when compared with carriers with no LOT gene. Tang et al. (2011) showed that urgent revascularization of CYP2C19*2 gene carriers was higher than in patients without the CYP2C19*2 gene by observing the prognosis of 267 patients undergoing 12-month clopidogrel therapy after PCI. Therefore, CYP2C19 polymorphisms have important clinical significance.

At present, there are a few of studies on the specific genotype of CYP2C19*3 and CYP2C19*2 in China. Zhang et al. (2014) showed that the frequencies of heterozygous and 
homozygous CYP2C19*2 was 44.1 and $10.4 \%$, respectively. Similarly, our study found that the frequencies of heterozygous and homozygous CYP2C19*2 was 50 and $10.26 \%$, respectively, in Zhengzhou city. There is no uniform standard for the definition of clopidogrel resistance and the results were empirically obtained by different detection methods. Gurbel et al. (2003) compared platelet aggregation after administration of clopidogrel, using base platelet aggregation based on ADP as an inducer, and proposed that the absolute difference $\leq 10 \%$ was regarded clopidogrel resistance. Müller et al. (2003) detected the inhibition rate of platelet aggregation (IPA) to define clopidogrel responsiveness, and 10\%, 10-30\%, and $\geq$ $30 \%$ IPA was defined as non-responsive, low response, and normal response, respectively. In this study, after pairwise comparisons of the platelet aggregation of each genotype of CYP2C19*3/*2, the results showed no significant differences in CC/CC, CT/CC, CC/CT (P $>0.05)$ and in TT/CC, CC/TT, CT/CT $(\mathrm{P}>0.05)$. Therefore, $\mathrm{CC} / \mathrm{CC}, \mathrm{CT} / \mathrm{CC}, \mathrm{CC} / \mathrm{CT}$ were regarded as normal metabolism of clopidogrel, while CC/TT, TT/CC, CT/CT were considered as clopidogrel resistant, requiring ticagrelor antiplatelet therapy instead of clopidogrel therapy. In this study, the frequency of wild-type homozygous CYP2C19*2/3 was $37.2 \%$ and the normal dose of clopidogrel could be used for these carriers. However, the frequency of the mutant was $62.8 \%$, of which the frequency of the single mutant heterozygote was $49.6 \%$. Combined with platelet aggregation, carriers of the wild-type homozygote and single mutant heterozygote of CYP2C19*2/3 should be given a routine dose of clopidogrel by oral administration. At present, only 1 patient suffered from thrombosis and there was no increase in the risk of in-stent thrombosis during the 3-12 month follow-up. Mega et al. (2011) showed that carriers of mutant heterozygote after administration of $225 \mathrm{mg} /$ day clopidogrel could obtain the equivalent inhibition level of patients with wild-type homozygote after taking 75 $\mathrm{mg} /$ day, but carriers with mutant homozygote, even if taking the maintenance dose of $300 \mathrm{mg} /$ day, could not achieve ideal platelet inhibition. This is based on the observation of vasodilatorstimulated phosphoprotein (VASP) - plateletreactivity index (PRI) and P2Y(12) reaction units (PRU)levels in mutant heterozygote and mutant homozygote carriers of CYP2C19 after administration of the maintenance dose of $75,150,225$, or $300 \mathrm{mg}$ clopidogrel. It was safe to give a conventional dose of clopidogrel for wild-type homozygote and single mutant heterozygote of CYP2C19*2/*3, but clopidogrel resistance should be monitored in patients with coronary heart disease to reduce the incidence of thrombosis. In this study, the frequency of double mutant heterozygote or single mutant homozygote of CYP2C19*2/*3 was $13.2 \%$ and no thrombotic events occurred after ticagrelor anti-platelet therapy. In addition, this study showed correlation between gender and genotype distribution $(\mathrm{P}>0.05)$ but no significant correlation between age and genotype $(\mathrm{P}<0.05)$. Li et al. (2014) showed no significant correlation between age and CYP1C19 genotype based on the observation of 252 male and 119 female cases. In that study, the sample size was small and the proportion of men in the selected patients was high; however, the incidence of coronary heart disease in males is significantly higher than in females. The study also showed that the mutation rate of CYP2C19*2 in a Zhengzhou population $(59.9 \%)$ was significantly higher than in Chinese $(30 \%)$ based on foreign statistics, while the mutation rate of CYP2C19*3 in a Zhengzhou population (5.6\%) was consistent with that in a Chinese population (5\%). Fontana et al. (2008) concluded that there was a correlation between CYP2C19*2 allele and clopidogrel resistance in patients visiting cardiovascular outpatient clinics.

Currently, there appears to be a causal association between genetic variant and 
clopidogrel resistance, and CYP2C19 gene polymorphisms might be only a manifestation type of the gene-decided loci. Therefore, it is unknown whether the polymorphisms of CYP2C19 directly affect the risk of cardiovascular events so further in-depth and genome-related studies are needed to solve these issues. Due to the small number of cases and short follow-up in this study, a long-term follow-up would be needed to study the outcome of carriers with mutant heterozygote and single mutant homozygote of CYP2C19*2 and CYP2C19*3 after oral administration of clopidogrel to guide clinical medication, improve prognosis, and reduce the economic burden to patients and society.

\section{Conflicts of interest}

The authors declare no conflict of interest.

\section{ACKNOWLEDGMENTS}

We would be thankful all the participants in this study.

\section{REFERENCES}

Collet JP, Hulot JS, Pena A, Villard E, et al. (2009). Cytochrome P450 2C19 polymorphism in young patients treated with clopidogrel after myocardial infarction: a cohort study. Lancet 373: 309-317. http://dx.doi.org/10.1016/S0140$\underline{6736(08) 61845-0}$

Fontana P, Senouf D and Mach F (2008). Biological effect of increased maintenance dose of clopidogrel in cardiovascular outpatients and influence of the cytochrome P450 2C19*2 allele on clopidogrel responsiveness. Thromb. Res. 121: 463-468. http://dx.doi.org/10.1016/j.thromres.2007.06.012

Gurbel PA, Bliden KP, Hiatt BL and O'Connor CM (2003). Clopidogrel for coronary stenting: response variability, drug resistance, and the effect of pretreatment platelet reactivity. Circulation 107: 2908-2913. http://dx.doi.org/10.1161/01. CIR.0000072771.11429.83

Hou X, Shi J and Sun H (2014). Gene polymorphism of cytochrome P450 2C19*2 and clopidogrel resistance reflected by platelet function assays: a meta-analysis. Eur. J. Clin. Pharmacol. 70: 1041-1047. http://dx.doi.org/10.1007/s00228$\underline{014-1714-\mathrm{X}}$

Jeong YH, Tantry US, Kim IS, Koh JS, et al. (2011). Effect of CYP2C19*2 and *3 loss-of-function alleles on platelet reactivity and adverse clinical events in East Asian acute myocardial infarction survivors treated with clopidogrel and aspirin. Circ. Cardiovasc. Interv. 4: 585-594. http://dx.doi.org/10.1161/CIRCINTERVENTIONS.111.962555

Kazui M, Nishiya Y, Ishizuka T, Hagihara K, et al. (2010). Identification of the human cytochrome P450 enzymes involved in the two oxidative steps in the bioactivation of clopidogrel to its pharmacologically active metabolite. Drug Metab. Dispos. 38: 92-99. http://dx.doi.org/10.1124/dmd.109.029132

Li PF, Yang HB, Zhao YT, Li L, et al. (2014). Polymorphism of clopidogrel metabolism-related gene CYP2C19 in patients with coronary artery disease. Chin. J. Pract. Med. 41: 64-65.

Liboredo R and Pena SD (2014). Pharmacogenomics: accessing important alleles by imputation from commercial genomewide SNP arrays. Genet. Mol. Res. 13: 5713-5721. http://dx.doi.org/10.4238/2014.July.25.27

Mega JL, Hochholzer W, Frelinger AL, 3rd, Kluk MJ, et al. (2011). Dosing clopidogrel based on CYP2C19 genotype and the effect on platelet reactivity in patients with stable cardiovascular disease. JAMA 306: 2221-2228. http://dx.doi. org/10.1001/jama.2011.1703

Müller I, Besta F, Schulz C, Massberg S, et al. (2003). Prevalence of clopidogrel non-responders among patients with stable angina pectoris scheduled for elective coronary stent placement. Thromb. Haemost. 89: 783-787.

Scott SA, Sangkuhl K, Stein CM, Hulot JS, et al.; Clinical Pharmacogenetics Implementation Consortium (2013). Clinical Pharmacogenetics Implementation Consortium guidelines for CYP2C19 genotype and clopidogrel therapy: 2013 update. Clin. Pharmacol. Ther. 94: 317-323. http://dx.doi.org/10.1038/clpt.2013.105

Severino G and Del Zompo M (2004). Adverse drug reactions: role of pharmacogenomics. Pharmacol. Res. 49: 363-373. http://dx.doi.org/10.1016/j.phrs.2003.05.003 
Sun B, Li J, Dong M, Yang L, et al. (2015). Diversity of platelet function and genetic polymorphism in clopidogrel-treated Chinese patients. Genet. Mol. Res. 14: 1434-1442. http://dx.doi.org/10.4238/2015.February.13.22

Tang XF, He C, Yuan JQ, Meng XM, et al. (2011). [Impact of cytochrome P450 2C19 polymorphisms on outcome of cardiovascular events in clopidogrel-treated Chinese patients after percutaneous coronary intervention]. Zhonghua. Xin Xue Guan Bing Za Zhi 39: 617-620.

Wedlund PJ (2000). The CYP2C19 enzyme polymorphism. Pharmacology 61: 174-183. http://dx.doi. org $/ 10.1159 / 000028398$

Zhang CL and Zhu N (2011). Review of the relationship between polymorphisms of cytochrome P450 $2 \mathrm{C} 19$ and clopidogrel responsiveness. Adv. Cardiovasc. Dis. 32: 858-862.

Zhang L, Chen Y, Jin Y, Qu F, et al. (2013). Genetic determinants of high on-treatment platelet reactivity in clopidogrel treated Chinese patients. Thromb. Res. 132: 81-87. http://dx.doi.org/10.1016/j.thromres.2013.05.006

Zhang XX, Yan LR, Wang DX, Han LL, et al. (2014). Association between CYP2C19 polymorphisms and clopidogrel pharmacodynamics in patients with coronary heart diseases undergoing PCI. Chin. J. New Drugs. 23: 67-71.

Zhou SF, Liu JP and Chowbay B (2009). Polymorphism of human cytochrome P450 enzymes and its clinical impact. Drug Metab. Rev. 41: 89-295. http://dx.doi.org/10.1080/03602530902843483 